\title{
Prognostic factors of brain metastasis and survival among HER2-positive metastatic breast cancer patients: a systematic literature review
}

Michelle D. Hackshaw ${ }^{1}$, Heather E. Danysh², Mackenzie Henderson ${ }^{3}$, Eric Wang ${ }^{3,4}$, Nora Tu ${ }^{3}$, Zahidul Islam³, Amy Ladner ${ }^{5}$, Mary E. Ritchey ${ }^{5}$ and Maribel Salas ${ }^{3,6^{*}}$ [D

\begin{abstract}
Background: Patients with breast cancer who overexpress the human epidermal growth factor receptor 2 (HER2) and subsequently develop brain metastasis (BM) typically experience poor quality of life and low survival. We conducted a comprehensive literature review to identify prognostic factors for BM and predictors of survival after developing BM, and the effects of therapies with different mechanisms of action among patients with HER2+ breast cancer (BC).

Methods: A prespecified search strategy was used to identify research studies investigating BM in patients with HER2+ BC published in English during January 1, 2009-to June 25, 2021. Articles were screened using a two-phase process, and data from selected articles were extracted.

Results: We identified 25 published articles including 4097 patients with HER2+ BC and BM. Prognostic factors associated with shorter time to BM diagnosis after initial BC diagnosis included younger age, hormone receptor negative status, larger tumor size or higher tumor grade, and lack of treatment with anti-HER2 therapy. Factors predictive of longer survival after BM included having fewer brain lesions ( $<3$ or a single lesion) and receipt of any treatment after BM, including radiosurgery, neurosurgery and/or systemic therapy. Patients receiving combination trastuzumab and lapatinib therapy or trastuzumab and pertuzumab therapy had the longest median survival compared with other therapies assessed in this review.

Conclusions: More research is needed to better understand risk factors for BM and survival after BM in the context of HER2+ BC, as well as the assessment of new anti-HER2 therapy regimens that may provide additional therapeutic options for BM in these patients.
\end{abstract}

Keywords: HER2-positive, HER2 + , Brain metastasis, Prognostic factors, Survival, Anti-HER2 therapy

\footnotetext{
* Correspondence: msalas@dsi.com

${ }^{3}$ Global Epidemiology Department, Daiichi Sankyo, Inc., 211 Mt. Airy Road, Basking Ridge, NJ 07920, USA

${ }^{6}$ CCEB/CPeRT, University of Pennsylvania Perelman School of Medicine,

Philadelphia, PA, USA

Full list of author information is available at the end of the article
}

(c) The Author(s). 2021 Open Access This article is licensed under a Creative Commons Attribution 4.0 International License, which permits use, sharing, adaptation, distribution and reproduction in any medium or format, as long as you give appropriate credit to the original author(s) and the source, provide a link to the Creative Commons licence, and indicate if changes were made. The images or other third party material in this article are included in the article's Creative Commons licence, unless indicated otherwise in a credit line to the material. If material is not included in the article's Creative Commons licence and your intended use is not permitted by statutory regulation or exceeds the permitted use, you will need to obtain permission directly from the copyright holder. To view a copy of this licence, visit http://creativecommons.org/licenses/by/4.0/. The Creative Commons Public Domain Dedication waiver (http://creativecommons.org/publicdomain/zero/1.0/) applies to the data made available in this article, unless otherwise stated in a credit line to the data. 


\section{Background}

Approximately 15 to $20 \%$ of patients with breast cancer (BC) have tumors with elevated levels of human epidermal growth factor receptor 2 (HER2), which are associated with an aggressive clinical phenotype and poor prognosis $[1,2]$. Up to $50 \%$ of patients with HER2-positive (HER2+) metastatic $\mathrm{BC}$ will develop brain metastasis (BM) during the course of the disease, often leading to worse morbidity and shorter survival [3]. Current treatment strategies for $\mathrm{BM}$ in patients with HER2+ $\mathrm{BC}$ incorporate local therapies and systemic therapies. Local therapies include surgery, whole-brain radiotherapy (WBRT), and stereotactic radiosurgery [4-6]. Systemic therapies include chemotherapy (e.g., docetaxel, capecitabine) and anti-HER2 therapies, which can encompass monoclonal antibodies such as trastuzumab (approved in the U.S. in 1998 [7]) and pertuzumab (2012 [8]), antibody-drug conjugates such as trastuzumab emtansine (2013 [9]), and small molecule tyrosine kinase inhibitors (TKIs) such as lapatinib (2007 [10]) and neratinib (2017 [11]) [4-6]. Recent evidence suggests that lapatinib and neratinib can penetrate the bloodbrain barrier (BBB), and therefore these drugs and similar HER2-targeting TKIs may be promising therapeutic options for patients $[12,13]$.

We conducted a literature review to assess the epidemiology of patients with HER2+ metastatic or advanced BC with $\mathrm{BM}$ by describing prognostic factors for developing $\mathrm{BM}$ and factors predictive of survival among patients with
BM. Additionally, differences in survival and time to progression by HER2-targeting treatment drug classes were explored based on the drug mechanism of action.

\section{Methods \\ Search design}

Electronic searches were conducted in PubMed and Embase. A predefined search strategy (Online Resource 1 and Online Resource 2) was used to identify research studies investigating BM in patients with HER2+ BC. The search was restricted to studies published in English. The original search was restricted to studies published during the period of January 1, 2009 to July 30, 2019. However, the protocol was amended to expand the search to June 25, 2021 to capture the most recent published literature. Titles and abstracts identified from the electronic databases were exported to an Excel (Microsoft Corporation; Redmond, Washington) file for screening. Systematic literature reviews and meta-analyses relevant to the study objectives were not themselves included within the scope of this literature review, but the bibliographies were reviewed to identify potential additional publications.

\section{Screening and extraction}

Articles were screened in a two-level process. In Level 1 screening, one researcher reviewed the titles and abstracts of the identified articles according to the literature review inclusion and exclusion criteria (Table 1)

Table 1 Inclusion and Exclusion Criteria for Level 1 (Titles and Abstracts) and Level 2 (Full-Text) Screening

\begin{tabular}{|c|c|c|}
\hline Criterion & Included & Excluded \\
\hline $\begin{array}{l}\text { Study } \\
\text { design }\end{array}$ & $\begin{array}{l}\text { - Randomized controlled trials } \\
\text { - Single-arm studies } \\
\text { - Observational research studies (e.g., prospective cohort study, retrospective database } \\
\text { study, cohort study, case-control study) } \\
\text { - Literature reviews and meta-analyses } \\
\text { - Natural history studies } \\
\text { - Incidence and prevalence studies } \\
\text { - Prognostic factor studies }\end{array}$ & $\begin{array}{l}\text { - Consensus reports } \\
\text { - Preclinical studies } \\
\text { - Nonsystematic reviews } \\
\text { - Case reports } \\
\text { - Case studies/series } \\
\text { - Editorials } \\
\text { - Commentaries } \\
\text { - Letters } \\
\text { - Guideline or position statements } \\
\text { - Economic analyses } \\
\text { - Animal or other nonhuman (e.g., bench) } \\
\text { studies } \\
\text { - Study of < } 25 \text { patients }\end{array}$ \\
\hline Population & $\begin{array}{l}\text { - Patients with diagnosis of metastatic, recurrent, advanced, incurable, or unresectable } \\
\text { HER2+ breast cancer (stages 3-4) with BM, either at the time of breast cancer diagnosis } \\
\text { or after breast cancer diagnosis }\end{array}$ & $\begin{array}{l}\text { - Aged under } 18 \text { y } \\
\text { - Only patients with HER2- or stage } 1 \text { or } 2 \\
\text { breast cancer } \\
\text { - Only patients with HER2+ breast cancer } \\
\text { without BM }\end{array}$ \\
\hline Treatment & $\begin{array}{l}\text { - Evaluation of chemotherapy by mechanism of action } \\
\text { - Nonpharmacological studies }\end{array}$ & $\begin{array}{l}\text { - Specific regimens of chemotherapy (not } \\
\text { mechanism of action) } \\
\text { - Patients receiving surgical or radiation } \\
\text { intervention in place of chemotherapy } \\
\text { - Patients receiving CDK } 4 / 6 \text { inhibitors }\end{array}$ \\
\hline Evaluation & $\begin{array}{l}\text { - Incidence or prevalence } \\
\text { - Prognostic and/or predictive factors } \\
\text { - Treatment outcomes (safety or effectiveness) }\end{array}$ & - PK/PD of treatments \\
\hline
\end{tabular}

BM brain metastasis; CDK4/6 cyclin-dependent kinase 4 and 6; HER2 human epidermal growth factor receptor 2; PD pharmacodynamics; PK pharmacokinetics

${ }^{a}$ Literature reviews and meta-analyses were not be included in the review but were used to identify primary studies not previously identified 
and selected articles for further review. In Level 2 screening, the full text of articles selected at Level 1 were reviewed by one researcher using the same set of inclusion and exclusion criteria. If there was any uncertainty about the inclusion of articles, the Level 2 reviewer discussed the article with a second researcher to confirm, by consensus, whether the article met the study inclusion and exclusion criteria. Data were extracted according to prespecified data fields using the full-text articles, including study population, country, observation period, sample size, and select sample characteristics. A checklist was used to assess the quality of the studies.

\section{Results}

The number of studies included and excluded at each stage of screening was documented in a PRISMA diagram (Fig. 1) [14]. The original search strategy yielded 232 records for Level 1 screening and the expanded search added 162 records for Level 1 screening, for a total of 394 records retrieved. The bibliographies of 8 systematic reviews/meta-analyses were reviewed and yielded one additional study to be included for full-text screening for a total of 138 articles that were included in the Level 2 full-text screening. A total of 25articles met the inclusion criteria described in Table 1 and were selected for data extraction.

\section{Study and patient population characteristics}

Online Resource 3 presents the study and patient characteristics of the 25studies included in this review. Overall, the studies covered more than 10,000 patients, of which 4097 patients had HER2+ BC with BM. Across all studies, the observation/enrollment period started as early as 1988 [15] and ended as late as 2020 [16]. Geographically, studies were conducted in Europe $(n=11)$, North America $(n=7)$, the Asia-Pacific region $(n=7)$, and Turkey $(n=1)$.

Among studies reporting the median age of patients at the time of their initial $\mathrm{BC}$ diagnosis $(n=19$ [76\%]), the median age range was 43 to 55 years. The sex distribution was typically not reported, although three studies did report including 100\% females [17-19]. Only two studies reported on race, which included $67 \%$ white patients [15] and 58\% white patients [20].

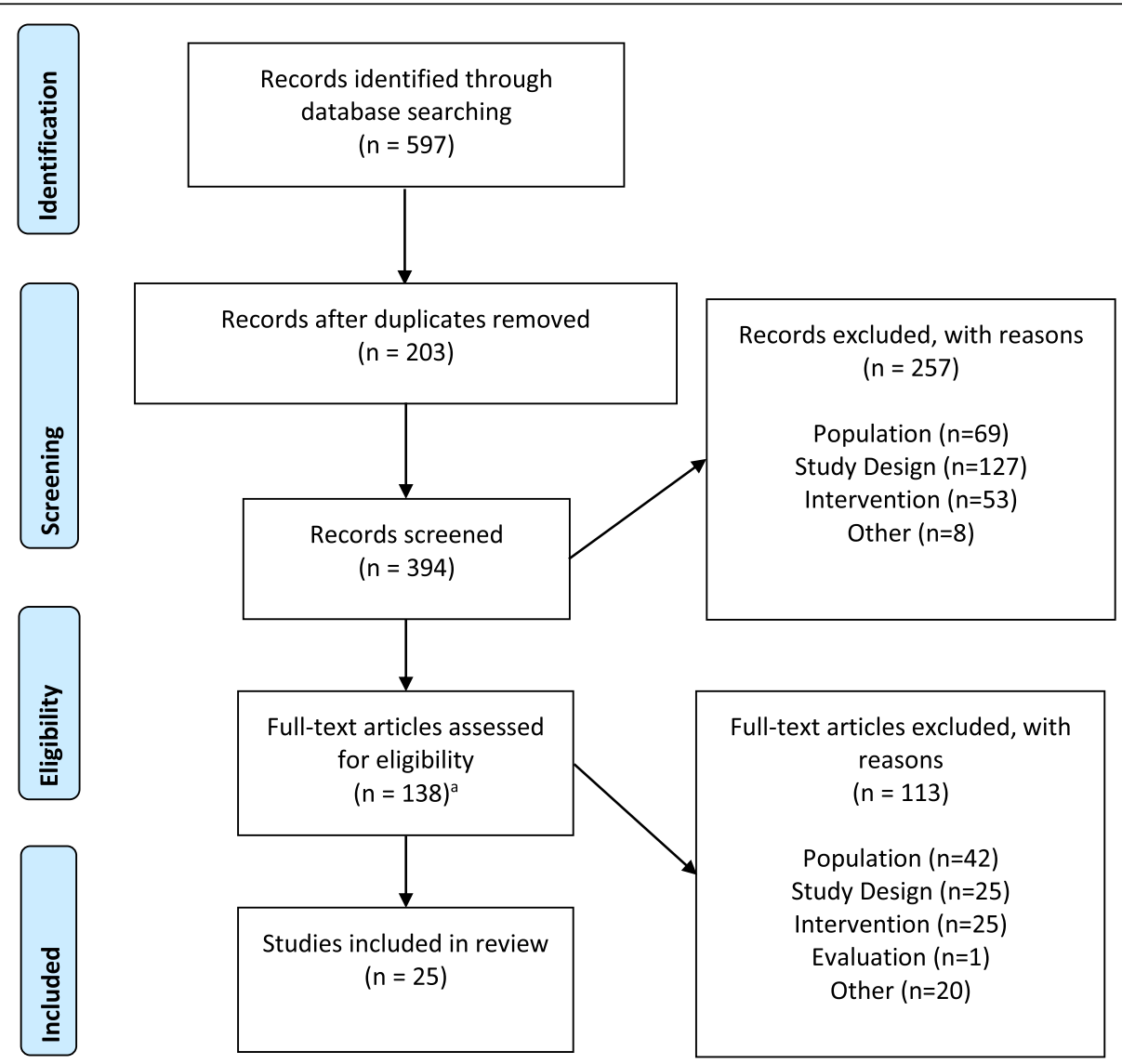

Fig. 1 PRISMA Diagram. ${ }^{a}$ This includes one additional article identified from the review of the bibliography of a systematic review/meta-analysis after initial record screening, hence there appears to be one extra article in this diagram; PRISMA = Preferred Reporting Items for Systematic Reviews and Meta-Analyses 
Twelve studies (48\%) included only patients with HER2+ status [18, 20-28], while the remaining studies $(n=13$ [52\%]) reported on patients in whom a subset were HER2+, ranging from $10.1 \%$ [29] to 58.2\% [30]. Among the seven studies that enrolled patients with or without BM [21-23, 26, 29, 31, 32], the prevalence of $\mathrm{BM}$ in patients with HER2+ BC ranged from 7.8\% [29] to $56.0 \%$ [23]. Twenty-one studies (84\%) provided information on hormone receptor (HR) status among patients with HER2+ BC, reporting a range of 24.2\% [33] to $71.0 \%$ [26] of patients with $\mathrm{HR}+$ status (i.e., estrogen receptor positive and/or progesterone receptor positive), while four studies (16\%) did not report on HR status $[16,30,34,35]$.

\section{Prognostic factors for developing brain metastasis}

Table 2 presents information on time to first BM diagnosis (TTBM) from initial HER2+ BC diagnosis and prognostic factors associated with a shorter TTBM. Across the 20 studies that reported information on TTBM, the shortest reported median TTBM was 10.8 months [31] and the longest was 76.2 months [26]. Among the 10 studies that reported on prognostic factors for BM diagnosis [15, 17, 18, 21-23, 26, 29-31], the most commonly assessed prognostic factors included age, HR status, receipt of anti-HER2 therapy, and tumor grade.

In three studies, age was not found to be associated with TTBM [21, 22, 29], while two studies reported an association between younger age at BC diagnosis (aged $\leq 40$ or $<50$ years in Maurer et al. [26] and Brufsky et al. [31], respectively) and shorter TTBM [26, 31]. Hormone receptor status was assessed as a prognostic factor for TTBM in eight studies; four studies reported no association between HR status and TTBM [21, 22, 26, 29], while four other studies reported that patients with HRstatus had a shorter TTBM compared with those who were $\mathrm{HR}+[15,23,30,31]$. Among the eight studies that assessed trastuzumab use and TTBM, five reported no association between receipt of trastuzumab and TTBM $[17,22,26,29,30]$, while three reported that patients who received trastuzumab had a longer TTBM compared with those who did not $[18,23,31]$. Two studies reported an association between higher tumor grade and shorter TTBM [21, 23], while two studies reported no association with tumor grade but did report an association between larger tumor size and shorter TTBM [26, 29].

Other treatment-related factors and TTBM were also assessed. In one study, longer TTBM was reported among those receiving treatment with endocrine therapy versus no endocrine therapy in the palliative care setting [30]. Conversely, another study reported that patients receiving adjuvant endocrine treatment had a shorter TTBM [26]. Longer TTBM was reported among patients who had surgery for their $\mathrm{BC}$ and among those treated with (neo) adjuvant anthracyclines + taxanes [26].

Other reported prognostic factors for shorter TTBM included higher disease burden (i.e., $\geq 2$ metastatic sites) [31], higher HER2 levels (measured by the HERmark ${ }^{\circ}$ Breast Cancer Assay) [21], distant relapse in $\leq 2$ years [22], first distant relapse at a visceral site [23], and nodal involvement [26]. One study reported on genetic factors of TTBM, but the reported associations in the discovery set were not observed in the validation set [23].

\section{Predictors of survival after brain metastasis among patients with HER2+ breast cancer}

Table 3 presents median time to death (i.e., survival) after BM diagnosis and predictive factors associated with shorter survival after BM diagnosis. Across the 23 studies that reported information on median survival after $\mathrm{BM}$, the shortest reported median survival was 5.2 months [34] and the longest was 28 months [21].

The most commonly assessed predictive factors for shorter survival after BM diagnosis included age, HR status, number of brain lesions, receipt of anti-HER2 therapy, and receipt of any systemic therapy. Four studies reported no association between age at BM diagnosis and survival $[15,27,31,35]$, three reported shorter survival among older patients $[18,24,33]$, and one reported shorter survival among younger patients [28]. The nine studies that observed HR status reported no association between HR status and survival after BM diagnosis [15, $24,25,27,28,31-33,36]$. Six studies reported that the presence of a higher number brain lesions compared with fewer brain lesions was associated with shorter survival after $\mathrm{BM}$ diagnosis [18, 24, 25, 27, 28, 33]. Other reported predictors of shorter survival after BM diagnosis included the presence of neurologic symptoms [24, 26, 27], tumor grade 3, two or more extracranial metastatic sites [33], central nervous system disease at BC diagnosis [31], uncontrolled extracranial metastases [27, 28], visceral metastases, and Radiation Therapy Oncology Group recursive partitioning analysis prognostic class 3 versus class $1 / 2$ [39].

While specific regimens were not assessed in this literature review, data were included from studies that did not assign treatment and for which any anti-HER2 treatment was captured within typical clinical practice. All 13 studies that assessed treatment with anti-HER2 therapy and survival after BM diagnosis reported an association between anti-HER2 therapy and survival. Twelve studies reported that patients who received anti-HER2 therapy after their BM diagnosis had a longer survival compared with patients who did not receive anti-HER2 therapy $[17-20,24,25,27,28,30,31,36,39]$. Four studies [18, $25,33,36]$ found that patients receiving both trastuzumab and lapatinib after their BM diagnosis had longer 


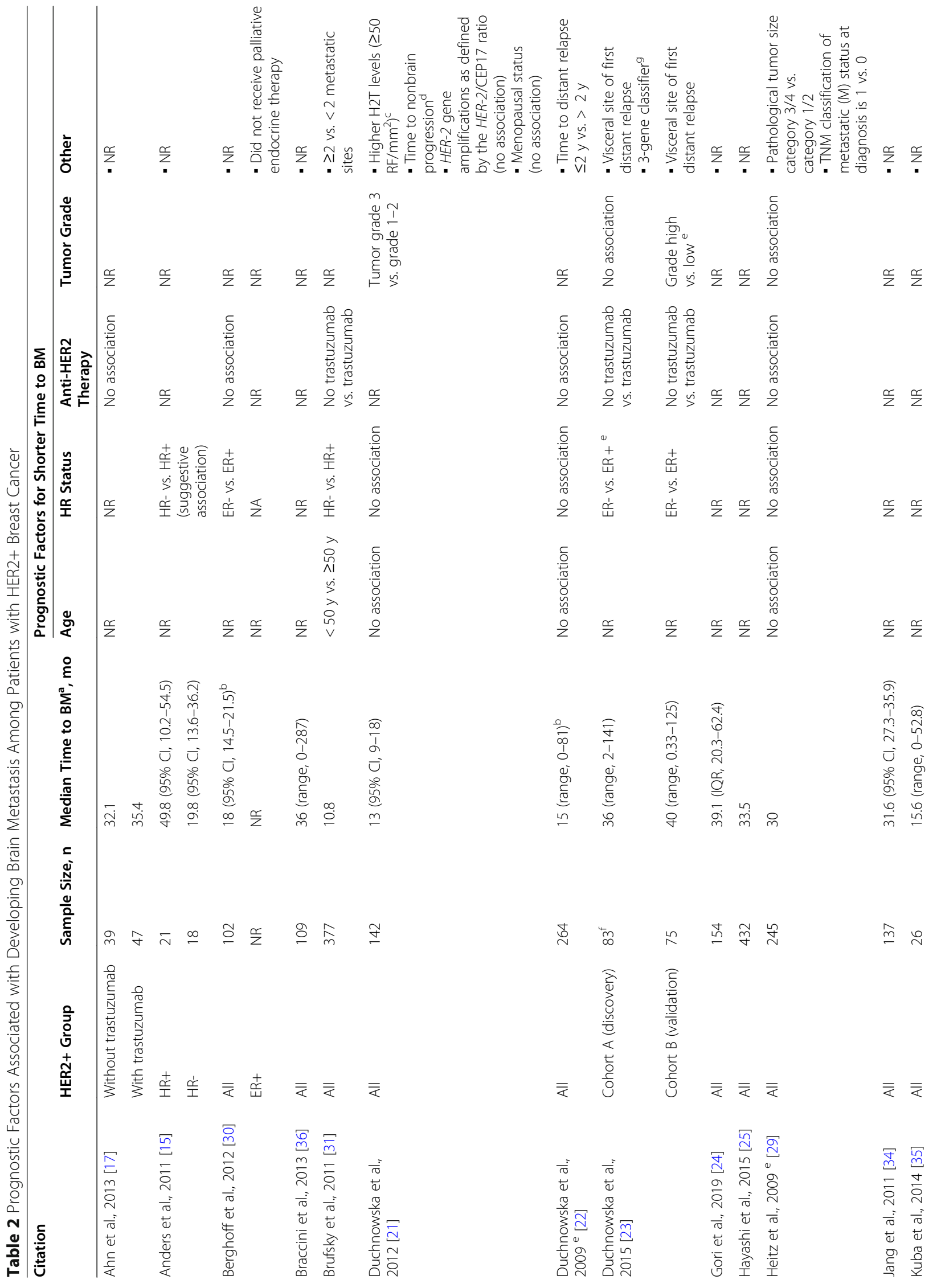




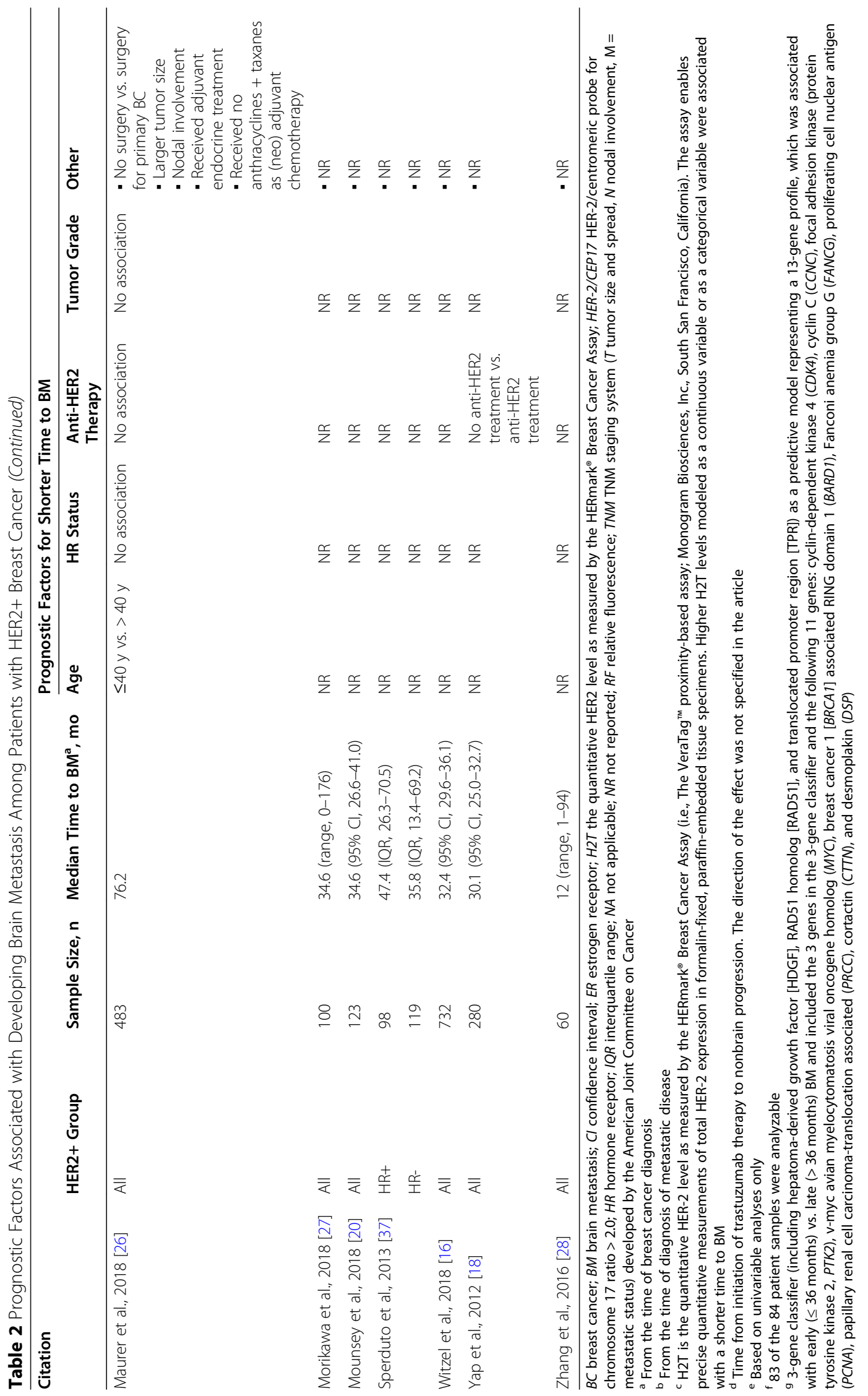




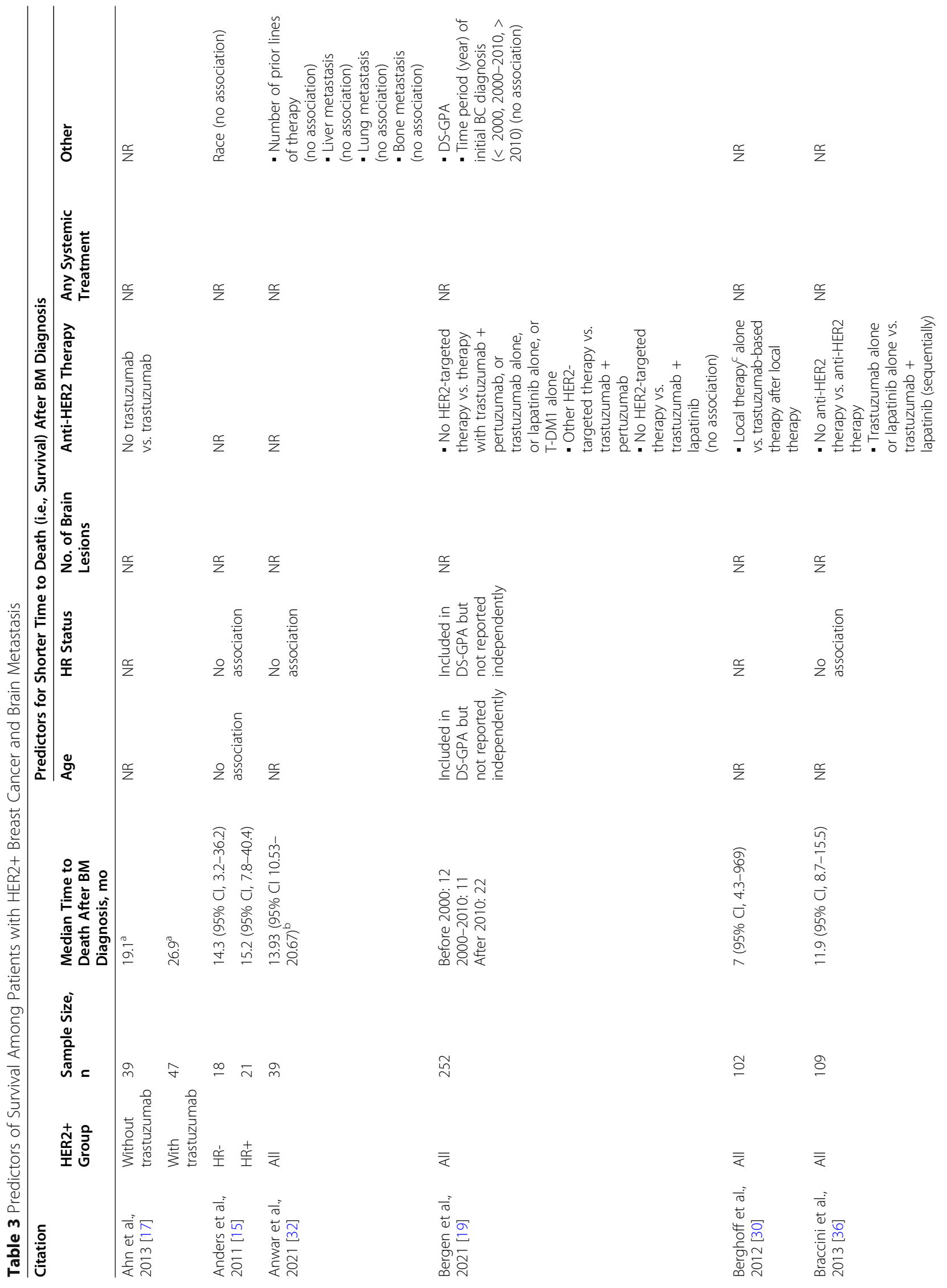




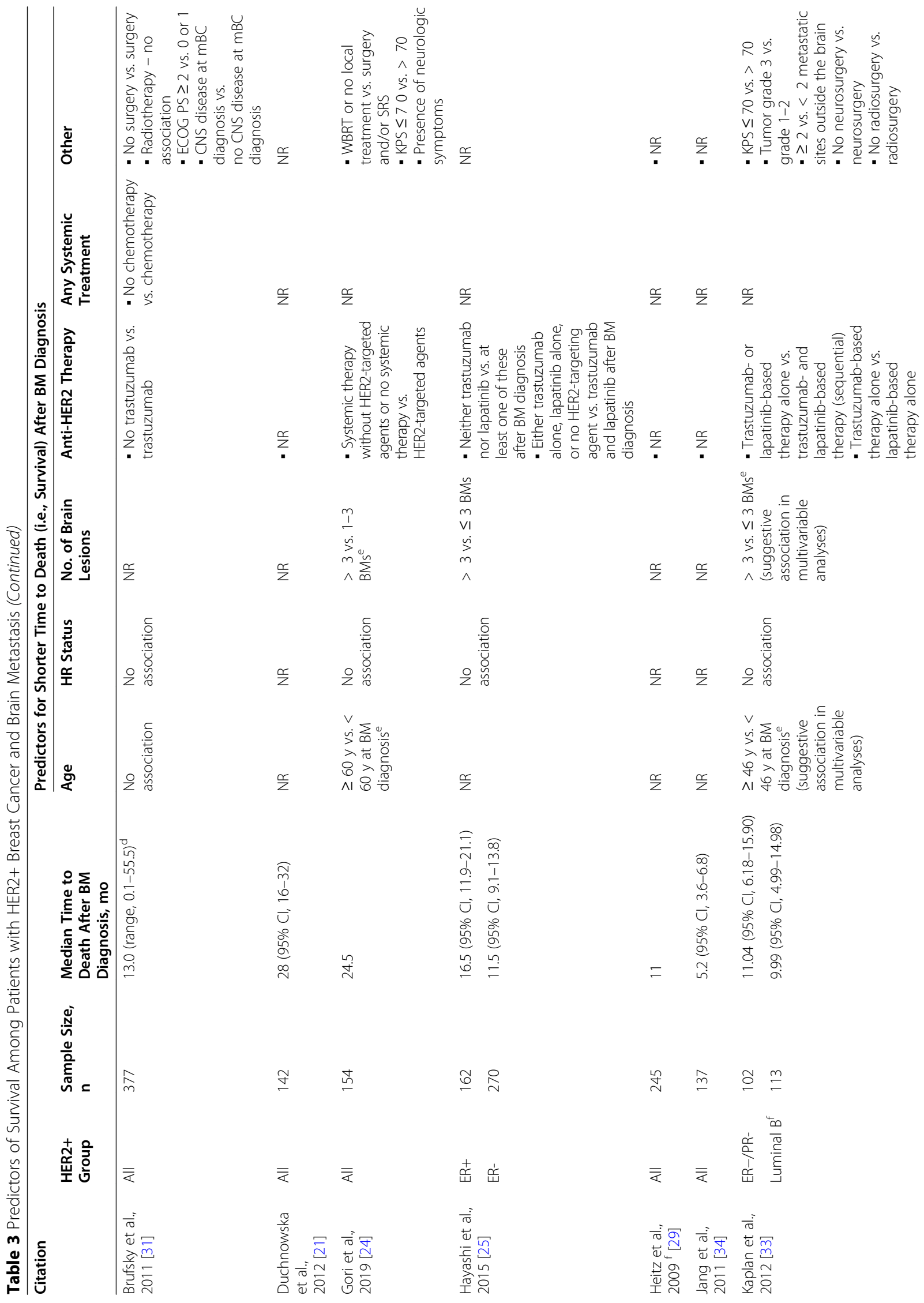




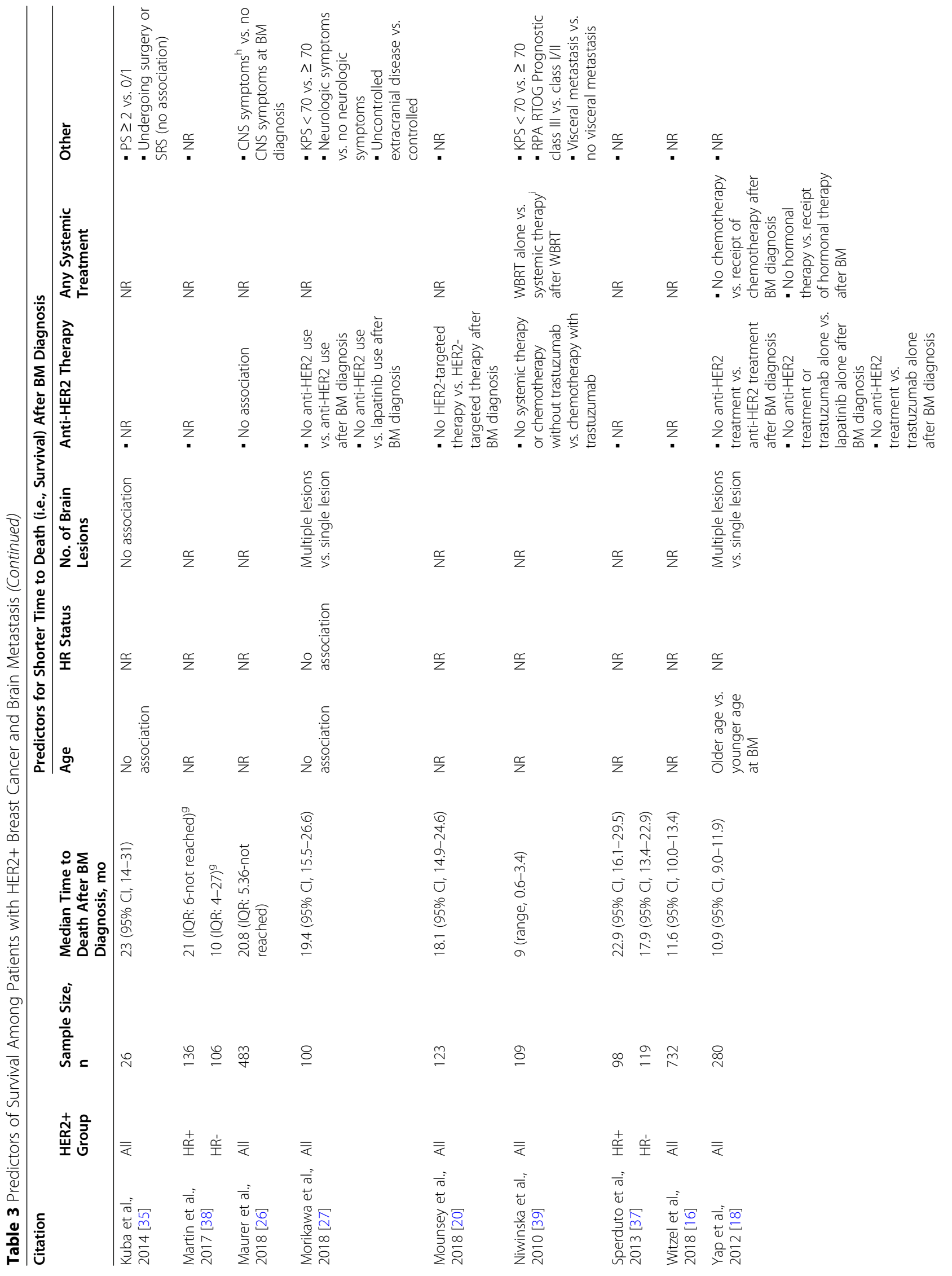




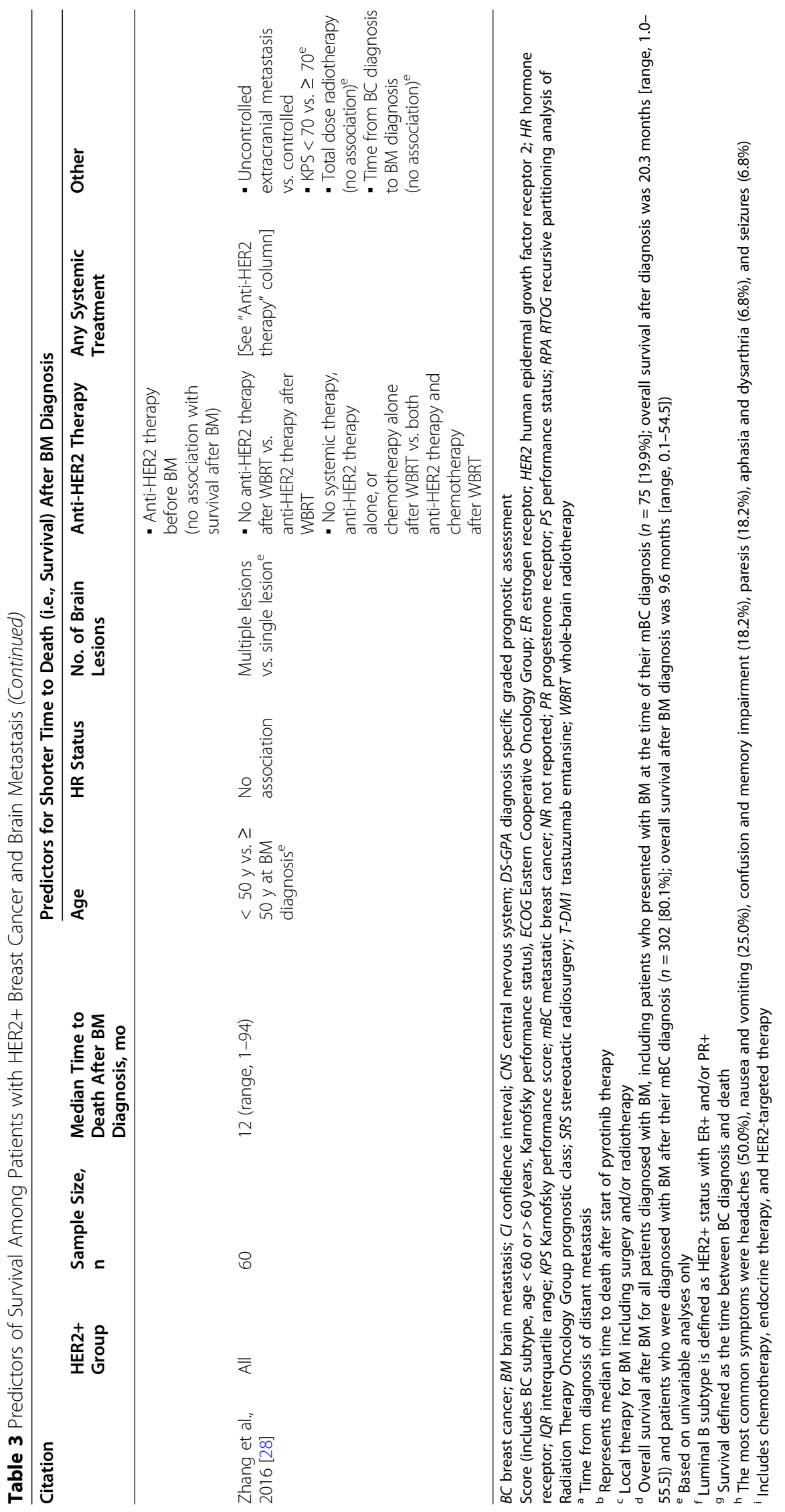


survival than those receiving either agent alone or no anti-HER2 therapy. One study found that patients receiving trastuzumab and pertuzumab after their BM diagnosis had longer survival than those receiving other HER2-targeted therapies or no HER2-targeted therapy [19].

Four studies [18, 31, 35, 39] assessed non-HER2targeted therapies and survival after BM diagnosis and reported that shorter survival was associated with no chemotherapy versus chemotherapy $[18,31]$, no hormonal therapy versus hormonal therapy [18], WBRT alone versus any systemic therapy after WBRT [39], and no systemic therapy versus any systemic therapy [35]. In addition, three studies reported that patients receiving surgery or stereotactic radiosurgery had longer survival than those not receiving these treatments [24, 31,33], while one study reported no association [35]. One study reported no association between the total dose of radiotherapy and survival after BM diagnosis [28].

\section{Treatment mechanism of action and outcomes after brain metastasis \\ HER2-targeted monoclonal antibodies}

Table 4 presents information on anti-HER2 treatment type by mechanism of action, and disease progression and survival after BM. Four studies evaluated survival among patients receiving a HER2-targeted monoclonal antibody (i.e., trastuzumab) after BM diagnosis and reported that overall survival (OS) was longer in patients who received trastuzumab after local therapy compared with patients who did not receive trastuzumab [17, 30, 31, 39]. In Ahn et al. [17], Berghoff et al. [30], and Brufsky et al. [31], the difference in median OS after BM between trastuzumab users and nonusers was 7.8 months, 10 months, and 13.8 months, respectively. Niwinska et al. [39] reported that among patients with $\mathrm{HR}+$ status, trastuzumab users had a 11-month longer median OS compared with nonusers $(P<0.001)$, and among patients with HR- status, trastuzumab users had a 6-month longer median OS compared with nonusers $(P=0.004)$. One study evaluated survival among patients receiving HER2-targeted monoclonal antibody combination therapy (i.e., trastuzumab + pertuzumab) and reported that OS was longer in patients who received trastuzumab + pertuzumab (44 months) compared to those who received other HER2-targeted therapy (17 months) or no HER2-targeted therapy (3 months) [19].

\section{HER2-targeted non-monoclonal antibodies}

One study evaluated survival among patients receiving a HER2-targeted non-monoclonal antibody (i.e., pyrotinib) with or without surgery/radiation, and reported that OS was longer in those with combination HER2-targeted non-monoclonal antibody and surgery/radiation [32].
OS was 20.7 months in those that received both a HER2-targeted non-monoclonal antibody (i.e., pyrotinib) and surgery/radiation, compared with 12.4 months in those who received only a HER2-targed non-monoclonal antibody (i.e., pyrotinib) $[P=0.021]$ [32].

\section{Tyrosine kinase inhibitor combination therapies}

Four studies evaluated the association between patients receiving lapatinib in combination with either trastuzumab or another anti-HER2 therapy, not otherwise specified [25, 27, 33, 36]. Braccini et al. [36] and Kaplan et al. [33] reported that patients receiving both lapatinib and trastuzumab had longer median OS (25.7 months and 23.6 months, respectively) after BM compared with those receiving either lapatinib alone or trastuzumab alone (9.6 months $[P<0.001]$ and 14.6 months $[P=0.023]$, respectively). Hayashi et al. [25] reported longer OS after $\mathrm{BM}$ in patients receiving lapatinib and trastuzumab compared with patients receiving only one of the two targeted therapies or no HER2-targeting therapy $(P<$ 0.001). Similarly, Morikawa et al. [27] reported that patients receiving lapatinib in combination with another anti-HER2 therapy, not otherwise specified, had lower mortality compared with those not receiving the combination therapy (adjusted hazard ratio $=0.26$; $95 \%$ confidence interval [CI], 0.13-0.52).

\section{Any anti-HER2 therapy}

The use of anti-HER2 therapy, not otherwise specified, after BM diagnosis was associated with an increase in OS in all studies except one [26]. Among the seven studies that reported longer survival in patients treated with anti-HER2 therapy after BM diagnosis [18-20, 24, 28, 33, 36], the median OS among those receiving anti-HER2 therapy ranged from 15.2 to 44 months compared with the median OS among those not receiving anti-HER2 therapy, which ranged from 3 to 13.8 months. Among patients receiving anti-HER2 therapy after BM diagnosis, median OS ranged from 11.8 [36] to 17.5 months [20] longer after their BM diagnosis compared with those not receiving anti-HER2 therapy. Conversely, Maurer et al. [26] reported no association between anti-HER2 therapy after BM diagnosis and OS.

Two studies reported on cerebral disease progression after BM diagnosis and treatment with anti-HER2 therapy $[26,36]$. Braccini et al. [36] reported longer cerebral progression-free survival in patients treated with antiHER2 therapy than in patients not receiving anti-HER2 therapy (6.3 months [ $95 \% \mathrm{CI}, 7.8-11.5]$ vs. 5.5 months [95\% CI, 1.2-6.7]), while Maurer et al. [26] reported no association between anti-HER2 therapy and a second central nervous system event. 
Table 4 Effect of Treatment Mechanisms of Action on Survival, Tumor Response, Time to Progression

\begin{tabular}{|c|c|c|}
\hline Citation & Therapy After BM & Outcome Assessed \\
\hline \multicolumn{3}{|c|}{ HER2-targeted monoclonal antibodies } \\
\hline $\begin{array}{l}\text { Ahn et al., } \\
2013[17]\end{array}$ & Trastuzumab & $\begin{array}{l}\text { OS after diagnosis of distant metastasis: trastuzumab, } 26.9 \text { mo; no } \\
\text { trastuzumab, } 19.1 \mathrm{mo} ; P=0.020\end{array}$ \\
\hline $\begin{array}{l}\text { Berghoff } \\
\text { et al., } 2012 \\
\text { [30] }\end{array}$ & Trastuzumab & $\begin{array}{l}\text { OS after BM diagnosis, } 7 \mathrm{mo}(95 \% \mathrm{Cl} \text {, 4.3-969); trastuzumab-based th } \\
\text { apy after completion of local therapy for BM (surgery, radiotherapy), } \\
\text { mo ( } 95 \% \mathrm{Cl}, 7.22-20.78) \text {; vs. not, } 4 \text { mo ( } 95 \% \mathrm{Cl}, 2.40-5.61)\end{array}$ \\
\hline $\begin{array}{l}\text { Brufsky } \\
\text { et al., } 2011 \\
\text { [31] }\end{array}$ & Trastuzumab & $\begin{array}{l}\text { OS after BM diagnosis, } 13.0 \text { mo (range, } 0.1-55.5) \text {; trastuzumab }{ }^{\mathrm{a}}, 17.5 \\
\text { vs. no trastuzumab, } 3.7 \text { mo; adjusted hazard ratio }=0.33(95 \% \mathrm{Cl}, 0.25 \\
\text { 0.46) }\end{array}$ \\
\hline $\begin{array}{l}\text { Niwinska } \\
\text { et al., } 2010 \\
{[39]}\end{array}$ & Trastuzumab & $\begin{array}{l}\text { OS after BM diagnosis } \\
\text { - HER2+/HR+: trastuzumab + chemotherapy after WBRT, } 13 \text { mo; } \\
\text { chemotherapy alone after WBRT, } 8 \text { mo; no systemic treatment after } \\
\text { WBRT, } 2 \text { mo; } P<0.001 \\
\text { - HER2+/HR-: trastuzumab + chemotherapy after WBRT, } 10 \text { mo; } \\
\text { chemotherapy alone after WBRT, } 8 \text { mo; no systemic treatment after } \\
\text { WBRT, } 4 \text { mo; } P=0.004\end{array}$ \\
\hline $\begin{array}{l}\text { Bergen } \\
\text { et al., } 2021 \\
{[19]}\end{array}$ & Trastuzumab + Pertuzumab & $\begin{array}{l}\text { OS after BM diagnosis, trastuzumab + pertuzumab, } 44 \text { mo; other HEF } \\
\text { targeted therapy, } 17 \text { mo; no HER2-targeted therapy, } 3 \text { mo }(P<0.001) \\
\text { Overall intracranial CBR, trastuzumab + pertuzumab as systemic first- } \\
\text { therapy after diagnosis of BM, } 100 \% \\
\text { Overall intracranial ORR, trastuzumab + pertuzumab as systemic first- } \\
\text { therapy after diagnosis of BM, } 92.9 \%\end{array}$ \\
\hline \multicolumn{3}{|c|}{ HER2-targeted non-monoclonal antibodies } \\
\hline $\begin{array}{l}\text { Anwar et al., } \\
2021[32]\end{array}$ & Pyrotinib & $\begin{array}{l}\text { OS after pyrotinib initiation, pyrotinib + surgery/radiation, } 20.7 \mathrm{mo} \text {; } \\
\text { pyrotinib only, } 12.4 \mathrm{mo}(P=0.021) \\
\text { PFS after pyrotinib initiation, pyrotinib + surgery/radiation, } 10.0 \mathrm{mo} \text {; } \\
\text { pyrotinib only, } 7.7 \mathrm{mo}(P=0.19) \\
\text { CBR after pyrotinib initiation, pyrotinib + surgery/radiation, } 58.6 \% \text {; } \\
\text { pyrotinib only, } 41.4 \% \\
\text { ORR after pyrotinib initiation pyrotinib + surgery/radiation, } 24.1 \% \text {; } \\
\text { pyrotinib only, } 31.0 \%\end{array}$ \\
\hline
\end{tabular}

\section{HER2-targeted monoclonal antibodies + Tyrosine kinase inhibitors}

Braccini Trastuzumab + Lapatinib

et al., 2013

[36]

Kaplan et al., Trastuzumab + Lapatinib

2012 [33]

Hayashi Trastuzumab + Lapatinib

et al., 2015

[25]
OS after BM diagnosis, 7 mo (95\% Cl, 4.3-969); trastuzumab-based ther(surgery, radiotherapy), 14

OS after BM diagnosis, $13.0 \mathrm{mo}$ (range, 0.1-55.5); trastuzumaba $17.5 \mathrm{mo}$ v. no trastuzumab, $3.7 \mathrm{mo}$; adjusted hazard ratio $=0.33(95 \% \mathrm{Cl}, 0.25-$

OS after BM diagnosis -

OS after BM diagnosis, trastuzumab + pertuzumab, 44 mo; other HER2therapy after diagnosis of BM, 100\%

Overall intracranial ORR, trastuzumab + pertuzumab as systemic first-line therapy after diagnosis of BM, $92.9 \%$

OS after BM diagnosis, trastuzumab and lapatinib (sequential), $25.7 \mathrm{mo}$ $(95 \% \mathrm{Cl}, 17.1-33.3)$; only 1 of the 2 targeted therapies, $9.6 \mathrm{mo}(95 \% \mathrm{Cl}$, 8.2-12.8); $P<0.001$

OS after BM diagnosis, trastuzumab- and lapatinib-based therapy (sequential), $23.6 \mathrm{mo}$; only 1 of the 2 targeted therapies, $14.6 \mathrm{mo} ; P=0.023$ OS after BM diagnosis, trastuzumab and lapatinib had a longer survival ${ }^{b}$ than trastuzumab alone, lapatinib alone, or no HER2-targeting agent; $P<$ 0.001

Tyrosine kinase inhibitors + other anti-HER2 therapies (not otherwise specified)

Morikawa Anti-HER2 therapy + Lapatinib

et al., 2018

[27]

\section{Anti-HER2 therapies (not otherwise specified)}

Braccini Anti-HER2 therapy

et al., 2013

[36]

Kaplan et al., HER2-targeted therapy (includes all patients receiving 2012 [33] trastuzumab, lapatinib, or both)

Gori et al., Anti-HER2 therapy

2019 [24]

Maurer Anti-HER2 treatment

et al., 2018
OS from BM diagnosis, 19.4 mo (95\% Cl, 15.5-26.6); anti-HER2 therapy with lapatinib vs. no use, adjusted hazard ratio $=0.26(95 \% \mathrm{Cl}, 0.13-0.52)$; anti-HER2 therapy without lapatinib vs. no use, adjusted hazard ratio = $0.32(95 \% \mathrm{Cl}, 0.18-0.59)$

- OS after BM diagnosis, 11.9 mo $(95 \% \mathrm{Cl}, 8.7-15.5)$; anti-HER2 therapy, $15.2 \mathrm{mo}(95 \% \mathrm{Cl}, 11.5-19.4)$; without anti-HER2 therapy, $3.4 \mathrm{mo}(95 \% \mathrm{Cl}$, $1.4-6)$

- Cerebral progression-free survival, anti-HER2 therapy, 6.3 mo $(95 \% \mathrm{Cl}$, 7.8-11.5); without anti-HER2 therapy, $5.5 \mathrm{mo}$ (95\% Cl, 1.2-6.7)

OS after BM diagnosis; HER2-targeted therapy, 16.7 mo; without HER2targeted therapy, $4.7 \mathrm{mo} ; P<0.001$

OS after BM diagnosis, 24.5 mo; HER2-targeted therapy (27.5 mo) vs. without anti-HER2 therapy (13.8 mo) (hazard ratio $=0.44[95 \% \mathrm{Cl}, 0.25-0.78]$ ) vs. no systemic therapy (2.1 mo) (hazard ratio $=0.09[95 \% \mathrm{Cl}, 0.05-0.16]$ )

No impact on the development of a second CNS event or on OS. OS, $20.8 \mathrm{mo}$ (IQR, 5.36-not reached) 
Table 4 Effect of Treatment Mechanisms of Action on Survival, Tumor Response, Time to Progression (Continued)

\begin{tabular}{|c|c|c|}
\hline Citation & Therapy After BM & Outcome Assessed \\
\hline \multicolumn{3}{|l|}{ [26] } \\
\hline $\begin{array}{l}\text { Mounsey } \\
\text { et al., } 2018 \\
{[20]}\end{array}$ & $\begin{array}{l}\text { HER2-targeted therapy (includes trastuzumab, lapatinib, } \\
\text { pertuzumab, and T-DM1) }\end{array}$ & $\begin{array}{l}\text { - Mortality after BM, receipt of HER2-targeted therapy after BM diagnosis, } \\
\text { adjusted hazard ratio }=0.61(95 \% \mathrm{Cl}, 0.39-0.97) \\
\text { - OS after BM diagnosis, } 18.1 \mathrm{mo}(95 \% \mathrm{Cl}, 14.9-24.6) \text {; HER2-targed therapy } \\
\text { ( } 62 \% \text { of patients), } 25.3 \mathrm{mo}(95 \% \mathrm{Cl}, 18.6-31.2) \text {; without HER2-targeted } \\
\text { therapy, } 7.8 \mathrm{mo}(95 \% \mathrm{Cl}, 4.56-15.0)\end{array}$ \\
\hline $\begin{array}{l}\text { Yap et al., } \\
2012[18]\end{array}$ & $\begin{array}{l}\text { Anti-HER2 therapy (includes trastuzumab alone, lapatinib } \\
\text { alone, or trastuzumab and lapatinib combined) }\end{array}$ & $\begin{array}{l}\text { OS after BM diagnosis, } 10.9 \mathrm{mo}(95 \% \mathrm{Cl}, 9.0-11.9) \text {; anti-HER2 therapy, } 18.5 \\
\text { mo; no anti-HER2 therapy, } 5.7 \text { mo; adjusted hazard ratio }=0.62(95 \% \mathrm{Cl} \text {, } \\
0.43-0.89)\end{array}$ \\
\hline $\begin{array}{l}\text { Zhang et al., } \\
2016[28]\end{array}$ & $\begin{array}{l}\text { Anti-HER2 therapy (includes trastuzumab alone, lapatinib } \\
\text { alone, or trastuzumab and lapatinib combined) }\end{array}$ & $\begin{array}{l}\text { OS after BM diagnosis, } 12 \text { mo (range, 1-94); anti-HER2 therapy after } \\
\text { WBRT, } 21 \text { mo, no anti-HER2 therapy after WBRT, } 9 \text { mo; } P=0.002\end{array}$ \\
\hline $\begin{array}{l}\text { Bergen et al., } \\
2021[19]\end{array}$ & HER2-targeted therapy, or no HER2-targeted therapy & $\begin{array}{l}\text { OS after BM diagnosis, other HER2-targeted therapy, } 17 \text { mo; no HER2- } \\
\text { targeted therapy, } 3 \text { mo }\end{array}$ \\
\hline
\end{tabular}

\section{Quality of studies}

Quality assessment of included studies was conducted using the Good Research for Comparative Effectiveness (GRACE) checklist [40, 41]. This 11-item scale contains 6 items related to quality of data and 5 items related to methodology. For each question, the quality is assessed based on "fit for purpose", and the quality is considered sufficient if the data or information provided per item is adequate for study purposes. The quality assessment revealed that all studies were eligible to be included in this review, even though 5 (20\%) studies have some limitations in the scientific methods (See Table 5).

\section{Discussion}

This literature review included 25 published articles that assessed a total of 4097patients with HER2+ BC with BM. Among these patients, prognostic factors of BM development and predictive factors of survival after BM diagnosis were assessed. Prognostic factors associated with shorter TTBM among patients with HER2+ BC included younger age at $\mathrm{BC}$ diagnosis, $\mathrm{HR}$ - versus $\mathrm{HR}+$ status $[15,23,30,31]$, no receipt of trastuzumab versus receipt of trastuzumab $[18,23,31]$, and higher tumor grade versus lower grades $[21,23]$. While these associations were observed across multiple studies, six studies found no association with TTBM and these prognostic factors $[17,21,22,26,29,30]$. Some studies reported longer TTBM in patients receiving trastuzumab or antiHER2 therapy, not otherwise specified, while some studies found no association.

Overall survival after BM diagnosis was shorter in patients with a higher number of lesions, was unaffected by HR status, and was variably affected by age at diagnosis. Treatment-related factors predictive of longer survival after BM included receipt of any systemic therapy.
Studies that assessed treatment with anti-HER2 therapy after BM diagnosis and survival $(n=13)$ reported that patients who received anti-HER2 therapy had longer survival after BM compared with patients who did not receive anti-HER2 therapy. Among studies that assessed survival differences between trastuzumab-based and lapatinib-based therapies $[18,25,33,36]$, patients receiving both trastuzumab and lapatinib after BM diagnosis had longer survival than those receiving either agent alone or no anti-HER2 therapy. One study found that patients receiving both trastuzumab and pertuzumab therapy after BM diagnosis had longer survival than those receiving other HER2-targeted therapy or no HER2-targeted therapy [19]. While trastuzumab has limited capability to cross the intact BBB, elevated concentrations of trastuzumab in the cerebrospinal fluid have been demonstrated when the BBB is impaired via radiotherapy and/or meningeal carcinomatosis [42]. As described in this review, trastuzumab in combination with lapatinib, which has been shown to cross the $\mathrm{BBB}$ in the BM setting [43], is favorable for survival in patients with BM. Lapatinib-based therapies may be an alternative therapeutic option for patients with $\mathrm{BM}$ and trastuzumab resistance [44]. The studies in this review also reported that survival after BM was improved with other therapies, including surgery or radiosurgery [24, 31, 33] and other systemic therapies [18, 31, 39]; however, the improvements in survival in patients receiving antiHER2 therapy superseded survival in those receiving one of these other therapies alone [24, 28, 39].

Contemporary patients with HER2+ $\mathrm{BC}$ have better OS compared with patients with HER2+ status who received treatment for $\mathrm{BC} 20$ years ago [45]. As more patients with HER2+ $\mathrm{BC}$ are living longer, with more opportunity to develop distant metastases, new 
Table 5 Quality of Studies Included

\begin{tabular}{|c|c|c|c|}
\hline Citation & Data Quality ${ }^{a}$ & Scientific Method $^{\text {b }}$ & Overall Quality Rating \\
\hline Anders et al., 2011 [15] & Sufficient & Sufficient & Sufficient \\
\hline Witzel et al., 2018 [16] & Sufficient & Sufficient & Sufficient \\
\hline Ahn et al., 2013 [17] & Sufficient & Insufficient & Sufficient \\
\hline Yap et al., 2012 [18] & Sufficient & Sufficient & Sufficient \\
\hline Mounsey et al., 2018 [20] & Sufficient & Sufficient & Sufficient \\
\hline Duchnowska et al., 2012 [21] & Sufficient & Insufficient & Sufficient \\
\hline Duchnowska et al., 2009 [22] & Sufficient & Insufficient & Sufficient \\
\hline Duchnowska et al., 2015 [23] & Sufficient & Sufficient & Sufficient \\
\hline Gori et al., 2019 [24] & Sufficient & Sufficient & Sufficient \\
\hline Hayashi et al., 2015 [25] & Sufficient & Sufficient & Sufficient \\
\hline Maurer et al., 2018 [26] & Sufficient & Sufficient & Sufficient \\
\hline Morikawa et al., 2018 [27] & Sufficient & Sufficient & Sufficient \\
\hline Zhang et al., 2016 [28] & Sufficient & Sufficient & Sufficient \\
\hline Heitz et al., $2009^{\text {e }}$ [29] & Sufficient & Sufficient & Sufficient \\
\hline Berghoff et al., 2012 [30] & Sufficient & Sufficient & Sufficient \\
\hline Brufsky et al., 2011 [31] & Sufficient & Sufficient & Sufficient \\
\hline Kaplan et al., 2012 [33] & Sufficient & Sufficient & Sufficient \\
\hline Jang et al., 2011 [34] & Sufficient & Insufficient & Sufficient \\
\hline Kuba et al., 2014 [35] & Sufficient & Sufficient & Sufficient \\
\hline Braccini et al., 2013 [36] & Sufficient & Sufficient & Sufficient \\
\hline Niwinska et al., 2010 [39] & Sufficient & Sufficient & Sufficient \\
\hline Sperduto et al., 2013 [37] & Sufficient & Insufficient & Sufficient \\
\hline Martin et al., 2017 [38] & Insufficient & Sufficient & Sufficient \\
\hline Anwar et al., 2021 [32] & Sufficient & Sufficient & Sufficient \\
\hline Bergen et al., 2021 [19] & Sufficient & Sufficient & Sufficient \\
\hline
\end{tabular}

a - Data attributes of exposure, outcomes (recording, objective measurement, validation) and important covariates/co-founders were assessed using 6 item checklist

b - Scientific methods were assessed using 5 item checklist and focused on the following areas

- New initiators of treatment

- Concurrent comparators

- Control of covariates/confounders/effect modifier

- Control of immortal time bias

- Analyses to evaluate the potential for bias for biased assessment

investigational agents are needed to treat patients with HER2+ BC with BM. One such drug is tucatinib, a nextgeneration small molecule TKI that is currently under assessment for improving outcomes specifically among patients with HER2+ BC with BM (ClinicalTrials.gov: NCT02614794 and NCT03975647) [46]. A recent randomized controlled trial found the addition of tucatinib compared with placebo to trastuzumab and capecitabine regimens improved progression-free survival and OS [47]. Trastuzumab deruxtecan, an antibody-drug conjugate, demonstrated encouraging antitumor activity during a phase 2 trial that evaluated patients with HER2+ metastatic BC, including 24 patients with BM [48]. Neratinib, a pan-HER TKI, showed efficacy in combination with capecitabine for treatment of refractory HER2+ BC with BM [49]. Treatment with anti-HER2 therapies, including current investigational and newly approved therapies, may provide additional options for patients with HER2+ BC with BM.

This review includes some limitations, such as the search was limited to the past 10 years and to Englishlanguage articles only. Only studies that assessed patients with $\mathrm{BM}$ are included in this review. Clinical trials often exclude patients with any BM or enroll only patients with clinically stable BM [50], limiting the ability to comprehensively assess the predictors of survival in patients with BM.

\section{Conclusion}

In this literature review, we describe the epidemiology of patients with HER2+ metastatic or advanced BC with $\mathrm{BM}$, including prognostic factors for developing BM, 
factors predictive of survival among patients with BM, and differences in survival and time to progression by HER2-targeting drug class based on drug mechanism of action. Prognostic factors associated with shorter TTBM included younger age, HR- status, no receipt of trastuzumab or anti-HER2 therapy, higher tumor grade, and larger tumor size. Predictors of longer OS after BM included receipt of anti-HER2 therapy or any systemic therapy, and the presence of fewer brain lesions $(<3$ or a single lesion). Trastuzumab and lapatinib combination therapy after BM diagnosis was associated with longer OS after BM compared with other treatments assessed in this review. More research is needed to better understand risk factors for BM and treatments that may improve outcomes.

\section{Abbreviations}

BBB: Blood-brain barrier; BC: Breast cancer; BM: Brain metastasis; HER2: Human epidermal growth factor receptor 2; HR: Hormone receptor; OS: Overall survival; TKI: Tyrosine kinase inhibitor; TTBM: Time to first brain metastasis diagnosis; WBRT: Whole-brain radiotherapy

\section{Supplementary Information}

The online version contains supplementary material available at https://doi. org/10.1186/s12885-021-08708-5.

\section{Additional file 1.}

\section{Acknowledgements}

The authors thank Brian Samsell of RTI Health Solutions for medical writing assistance.

\section{Authors' contributions}

$\mathrm{MDH}$ and MS: Conceptualized, designed the study, interpreted the data and substantively revised the manuscript. MH and EW: Reviewed, designed the updated study, interpreted the data and revised the manuscript. HED, AL and MER: Acquired and analyzed data, drafted the manuscript. NT and Zl: Designed the study, interpreted the data and substantively revised the manuscript. All authors read and approved the submitted version the manuscript and agreed both to be personally accountable for the author's own contributions and the accuracy or integrity of any part of the work.

\section{Funding}

Financial support for the study was provided by Daiichi Sankyo, Inc. RTI Health Solutions received funding under a research contract with Daiichi Sankyo, Inc. to conduct this study and provide editorial support in the form of manuscript writing, styling, and submission.

\section{Availability of data and materials}

Data has been made available as electronic supplementary material.

\section{Declarations}

Ethics approval and consent to participate

The article does not contain any studies with human participants or animals performed by any of the authors, and the protocol required waiver from IRB. As this study is based on a publicly available database without identifying patient information, informed consent was not required.

\section{Consent for publication}

Not applicable.

\section{Competing interests}

MS, MDH, MH, NT, and ZI are Daiichi Sankyo, Inc. employees. EW is an employee of Rutgers University and is contracted to Daiichi Sankyo, Inc. HED,
AL, and MER are employees of RTI Health Solutions, an independent nonprofit research organization that performs contracted project work for medical device and pharmaceutical companies.

\section{Author details}

${ }^{1}$ Global Medical Affairs, Daiichi Sankyo, Basking Ridge, NJ, USA. ${ }^{2}$ Department of Epidemiology, RTI Health Solutions, Waltham, MA, USA. ${ }^{3}$ Global Epidemiology Department, Daiichi Sankyo, Inc., 211 Mt. Airy Road, Basking Ridge, NJ 07920, USA. ${ }^{4}$ Rutgers Institute for Pharmaceutical Industry Fellowships, Piscataway, NJ, USA. ${ }^{5}$ Department of Epidemiology, RTI Health Solutions, Research Triangle Park, NC, USA. ${ }^{6}$ CCEB/CPeRT, University of Pennsylvania Perelman School of Medicine, Philadelphia, PA, USA.

Received: 6 October 2020 Accepted: 2 August 2021

Published online: 28 August 2021

\section{References}

1. Howlader N, Altekruse SF, Li Cl, Chen WW, Clarke CA, Ries LAG, et al. US incidence of breast cancer subtypes defined by joint hormone receptor and HER2 status. J Natl Cancer Inst. 2014;106(5). https://doi.org/10.1093/jnci/ dju055.

2. Goddard KA, Weinmann S, Richert-Boe K, Chen C, Bulkley J, Wax C. HER2 evaluation and its impact on breast cancer treatment decisions. Public Health Genomics. 2012;15(1):1-10. https://doi.org/10.1159/000325746.

3. Leone JP, Lin NU. Systemic therapy of central nervous system metastases of breast cancer. Curr Oncol Rep. 2019;21(6):49. https://doi.org/10.1007/s11912019-0802-6.

4. National Comprehensive Cancer Network. NCCN clinical practice guidelines in oncology (NCCN guidelines): breast Cancer. 2019. Available at: https://www.nccn.org/professionals/physician_gls/pdf/breast. pdf. Accessed: 1 Jul 2019

5. Ramakrishna N, Temin S, Lin NU. Recommendations on disease management for patients with advanced human epidermal growth factor receptor 2-positive breast cancer and brain metastases: ASCO clinical practice guideline update summary. J Oncol Pract. 2018;14(8):505-7. https:// doi.org/10.1200/JOP.18.00291.

6. Cardoso F, Senkus E, Costa A, Papadopoulos E, Aapro M, André F, et al. 4th ESO-ESMO international consensus guidelines for advanced breast Cancer (ABC 4)dagger. Ann Oncol. 2018;29(8):1634-57. https://doi.org/10.1093/a nnonc/mdy192.

7. US Food and Drug Administration. Herceptin prescribing information. 2018. Available at: https://www.accessdata.fda.gov/drugsatfda_docs/label/2018/1 03792s5345lbl.pdf. Accessed: 4 Nov 2019.

8. US Food and Drug Administration. Perjeta prescribing information. 2018 Available at: https://www.accessdata.fda.gov/drugsatfda_docs/label/2018/12 5409s123lbl.pdf. Accessed: 4 Nov 2019.

9. US Food and Drug Administration. Kadcyla prescribing information. 2019. Available at: https://www.accessdata.fda.gov/drugsatfda_docs/label/2019/12 5427s105lbl.pdf. Accessed: 28 Jun 2019.

10. US Food and Drug Administration. Tykerb prescribing information. 2018. Available at: https://www.accessdata.fda.gov/drugsatfda_docs/label/2018/ 022059s024lbl.pdf. Accessed: 14 Nov 2019.

11. US Food and Drug Administration. Nerlynx prescribing information. 2017. Available at: https://www.accessdata.fda.gov/drugsatfda_docs/label/2017/2 08051s000lbl.pdf. Accessed: 11 Jul 2019.

12. Kotecki N, Gombos A, Awada A. Adjuvant therapeutic approaches of HER2positive breast cancer with a focus on neratinib maleate. Expert Rev Anticancer Ther. 2019;19(6):447-54. https://doi.org/10.1080/14737140.2019.1 613892.

13. Laakmann E, Muller V, Schmidt M, Witzel I. Systemic treatment options for HER2-positive breast cancer patients with brain metastases beyond trastuzumab: a literature review. Breast Care (Basel). 2017;12(3):168-71. https://doi.org/10.1159/000467387.

14. Moher D, Liberati A, Tetzlaff J, Altman DG, Group P. Preferred reporting items for systematic reviews and meta-analyses: the PRISMA statement. PLoS Med. 2009;6(7):e1000097. https://doi.org/10.1371/journal.pmed.1 000097.

15. Anders CK, Deal AM, Miller CR, Khorram C, Meng H, Burrows E, et al. The prognostic contribution of clinical breast cancer subtype, age, and race among patients with breast cancer brain metastases. Cancer. 2011;117(8): 1602-11. https://doi.org/10.1002/cncr.25746. 
16. Witzel I, Laakmann E, Weide R, et al. Treatment and outcomes of patients in the brain metastases in breast Cancer Network registry. Eur J Cancer. 2018;102:1.

17. Ahn HK, Park YH, Lee SJ, Park S, Maeng CH, Park W, et al. Clinical implication of time to brain metastasis (TTBM) according to breast cancer subtypes. Springerplus. 2013;2(1):136. https://doi.org/10.1186/2193-1801-2-136.

18. Yap YS, Cornelio GH, Devi BC, et al. Brain metastases in Asian HER2-positive breast cancer patients: anti-HER2 treatments and their impact on survival. $\mathrm{Br}$ J Cancer. 2012;107(7):1075-82. https://doi.org/10.1038/bjc.2012.346.

19. Bergen ES, Binter A, Starzer AM, et al. Favourable outcome of patients with breast cancer brain metastases treated with dual HER2 blockade of trastuzumab and pertuzumab. Ther Adv Med Oncol. 2021;13: 17588359211009002.

20. Mounsey LA, Deal AM, Keith KC, Benbow JM, Shachar SS, Zagar T, et al. Changing natural history of HER2-positive breast cancer metastatic to the brain in the era of new targeted therapies. Clin Breast Cancer. 2018;18(1): 29-37. https://doi.org/10.1016/j.clbc.2017.07.017.

21. Duchnowska R, Biernat W, Szostakiewicz B, Sperinde J, Piette F, Haddad M, et al. Correlation between quantitative HER-2 protein expression and risk for brain metastases in HER-2+ advanced breast cancer patients receiving trastuzumab-containing therapy. Oncologist. 2012;17(1):26-35. https://doi. org/10.1634/theoncologist.2011-0212.

22. Duchnowska R, Dziadziuszko R, Czartoryska-Arlukowicz B, et al. Risk factors for brain relapse in HER2-positive metastatic breast cancer patients. Breast Cancer Res Treat. 2009;117(2):297-303. https://doi.org/10.1007/s1 0549-008-0275-z.

23. Duchnowska R, Jassem J, Goswami CP, Dundar M, Gökmen-Polar Y, Li L, et al. Predicting early brain metastases based on clinicopathological factors and gene expression analysis in advanced HER2-positive breast cancer patients. J Neuro-Oncol. 2015;122(1):205-16. https://doi.org/10.1007/s11060014-1704-y.

24. Gori S, Puglisi F, Moroso S, Fabi A, la Verde N, Frassoldati A, et al. The HERBA study: a retrospective multi-institutional Italian study on patients with brain metastases from HER2-positive breast cancer. Clin Breast Cancer. 2019;19(4): e501-10. https://doi.org/10.1016/j.clbc.2019.05.006.

25. Hayashi N, Niikura N, Masuda N, Takashima S, Nakamura R, Watanabe Kl, et al. Prognostic factors of HER2-positive breast cancer patients who develop brain metastasis: a multicenter retrospective analysis. Breast Cancer Res Treat. 2015;149(1):277-84. https://doi.org/10.1007/s10549-014-3237-7.

26. Maurer C, Tulpin L, Moreau M, Dumitrescu C, de Azambuja E, Paesmans M, et al. Risk factors for the development of brain metastases in patients with HER2-positive breast cancer. ESMO Open. 2018;3(6):e000440. https://doi. org/10.1136/esmoopen-2018-000440.

27. Morikawa A, Wang R, Patil S, Diab A, Yang J, Hudis CA, et al. Characteristics and prognostic factors for patients with HER2-overexpressing breast cancer and brain metastases in the era of HER2-targeted therapy: an argument for earlier detection. Clin Breast Cancer. 2018;18(5):353-61. https://doi.org/10.1 016/j.clbc.2017.12.009.

28. Zhang Q, Chen J, Yu X, Cai G, Yang Z, Cao L, et al. Survival benefit of antiHER2 therapy after whole-brain radiotherapy in HER2-positive breast cancer patients with brain metastasis. Breast Cancer. 2016;23(5):732-9. https://doi. org/10.1007/s12282-015-0631-X.

29. Heitz F, Harter P, Lueck HJ, Fissler-Eckhoff A, Lorenz-Salehi F, Scheil-Bertram $\mathrm{S}$, et al. Triple-negative and HER2-overexpressing breast cancers exhibit an elevated risk and an earlier occurrence of cerebral metastases. Eur J Cancer. 2009;45(16):2792-8. https://doi.org/10.1016/j.ejca.2009.06.027.

30. Berghoff A, Bago-Horvath Z, De Vries C, et al. Brain metastases free survival differs between breast cancer subtypes. Br J Cancer. 2012;106(3):440-6. https://doi.org/10.1038/bjc.2011.597.

31. Brufsky AM, Mayer M, Rugo HS, Kaufman PA, Tan-Chiu E, Tripathy D, et al. Central nervous system metastases in patients with HER2-positive metastatic breast cancer: incidence, treatment, and survival in patients from registHER. Clin Cancer Res. 2011;17(14):4834-43. https://doi.org/10.1158/1 078-0432.CCR-10-2962.

32. Anwar M, Chen Q, Ouyang D, Wang S, Xie N, Ouyang Q, et al. Pyrotinib treatment in patients with HER2-positive metastatic breast Cancer and brain metastasis: exploratory final analysis of real-world. Multicenter Data Clin Cancer Res. 2021;27(16):4634-41. https://doi.org/1 0.1158/1078-0432.CCR-21-0474.

33. Kaplan MA, Isikdogan A, Koca D, Kucukoner M, Gumussoy O, Yildiz R, et al. Biological subtypes and survival outcomes in breast cancer patients with brain metastases (study of the Anatolian Society of Medical Oncology). Oncology. 2012;83(3):141-50. https://doi.org/10.1159/000338782.
34. Jang G, Lee SS, Ahn JH, Jung KH, Lee H, Gong G, et al. Clinical features and course of brain metastases in triple-negative breast cancer: comparison with human epidermal growth factor receptor 2-positive and other type at single institution in Korea. Breast Cancer Res Treat. 2011;128(1):171-7. https://doi.org/10.1007/s10549-011-1526-y.

35. Kuba S, Ishida M, Nakamura Y, Yamanouchi K, Minami S, Taguchi K, et al. Treatment and prognosis of breast cancer patients with brain metastases according to intrinsic subtype. Jpn J Clin Oncol. 2014;44(11):1025-31. https://doi.org/10.1093/jjco/hyu126.

36. Braccini AL, Azria D, Thezenas S, Romieu G, Ferrero JM, Jacot W. Prognostic factors of brain metastases from breast cancer: impact of targeted therapies. Breast. 2013;22(5):993-8. https://doi.org/10.1016/j.breast.2013.05.011.

37. Sperduto PW, Kased N, Roberge D, Chao ST, Shanley R, Luo X, et al. The effect of tumor subtype on the time from primary diagnosis to development of brain metastases and survival in patients with breast cancer. J Neuro-Oncol. 2013;112(3):467-72. https://doi.org/10.1007/s11060-013-1083-9.

38. Martin AM, Cagney DN, Catalano PJ, Warren LE, Bellon JR, Punglia RS, et al. Brain metastases in newly diagnosed breast cancer: a population-based study. JAMA Oncol. 2017;3(8):1069-77. https://doi.org/10.1001/jamaoncol.2 017.0001.

39. Niwinska A, Murawska M, Pogoda K. Breast cancer brain metastases: differences in survival depending on biological subtype, RPA RTOG prognostic class and systemic treatment after whole-brain radiotherapy (WBRT). Ann Oncol. 2010;21(5):942-8. https://doi.org/10.1 093/annonc/mdp407.

40. Dreyer NA, Bryant A, Velentgas P. The GRACE checklist: a validated assessment tool for high quality observational studies of comparative effectiveness. J Manag Care Spec Pharm. 2016;22(10):1107-13. https://doi. org/10.18553/jmcp.2016.22.10.1107.

41. Dreyer NA, Velentgas $P$, Westrich $K$, Dubois $R$. The GRACE checklist for rating the quality of observational studies of comparative effectiveness: a tale of Hope and caution. J Manag Care Pharm. 2014;20(3):301-8. https://doi.org/1 0.18553/jmcp.2014.20.3.301.

42. Stemmler HJ, Schmitt M, Willems A, Bernhard H, Harbeck N, Heinemann V. Ratio of trastuzumab levels in serum and cerebrospinal fluid is altered in HER2-positive breast cancer patients with brain metastases and impairment of blood-brain barrier. Anti-Cancer Drugs. 2007;18(1):23-8. https://doi.org/1 0.1097/01.cad.0000236313.50833.ee.

43. Saleem A, Searle GE, Kenny LM, Huiban M, Kozlowski K, Waldman AD, et al. Lapatinib access into normal brain and brain metastases in patients with her-2 overexpressing breast cancer. EJNMMI Res. 2015;5(1):30. https://doi. org/10.1186/s13550-015-0103-5.

44. Lin NU, Dieras V, Paul D, et al. Multicenter phase II study of lapatinib in patients with brain metastases from HER2-positive breast cancer. Clin Cancer Res. 2009; 15(4):1452-9. https:/doi.org/10.1158/1078-0432.CCR-08-1080.

45. Pernas S, Tolaney SM. HER2-positive breast cancer: new therapeutic frontiers and overcoming resistance. Ther Adv Med Oncol. 2019;11: 1758835919833519

46. Murthy R, Borges VF, Conlin A, Chaves J, Chamberlain M, Gray T, et al. Tucatinib with capecitabine and trastuzumab in advanced HER2-positive metastatic breast cancer with and without brain metastases: a nonrandomised, open-label, phase 1b study. Lancet Oncol. 2018;19(7):880-8. https://doi.org/10.1016/S1470-2045(18)30256-0.

47. Murthy RK, Loi S, Okines A, et al. Tucatinib, Trastuzumab, and Capecitabine for HER2-positive metastatic breast Cancer. N Engl J Med. 2019.

48. Modi S, Saura C, Yamashita T, et al. Trastuzumab Deruxtecan in previously treated HER2-positive breast Cancer. N Engl J Med. 2019.

49. Freedman RA, Gelman RS, Anders CK, Melisko ME, Parsons HA, Cropp AM, et al. TBCRC 022: a phase II trial of Neratinib and Capecitabine for patients with human epidermal growth factor receptor 2-positive breast Cancer and brain metastases. J Clin Oncol. 2019;37(13):1081-9. https://doi.org/10.1200/ JCO.18.01511.

50. Kim ES, Bruinooge SS, Roberts S, Ison G, Lin NU, Gore L, et al. Broadening eligibility criteria to make clinical trials more representative: American Society of Clinical Oncology and friends of Cancer research joint research statement. J Clin Oncol. 2017;35(33):3737-44. https://doi.org/10.1200/JCO.2 017.73 .7916

\section{Publisher's Note}

Springer Nature remains neutral with regard to jurisdictional claims in published maps and institutional affiliations. 